\title{
Lumbar Cryptococcal Osteomyelitis Mimicking Metastatic Tumor
}

\author{
Hyun Seok Joo, Jung-Ki Ha, Chang Ju Hwang, Dong-Ho Lee, Choon Sung Lee, Jae Hwan Cho \\ Department of Orthopedic Surgery, Asan Medical Center, University of Ulsan College of Medicine, Seoul, Korea
}

Cryptococcus is an encapsulated, yeast-like fungus that rarely causes infection in immunocompetent patients. We present the case report of a 66-year-old female patient with a history of rectal cancer with an isolated lumbar vertebral cryptococcosis proven by biopsy performed during operation. The patient was not an immunocompromised host and did not have any other risk factors except the history of cured rectal cancer. The presumptive diagnosis based on imaging studies was metastatic spine cancer, so operation was performed. However, cryptococcal osteomyelitis was diagnosed in the pathologic examination. This case report emphasizes that we should be aware that lumbar cryptococcosis can be a rare cause of mimicking lesions with metastatic cancer.

Keywords: Lumbar vertebrae; Cryptococcus; Osteomyelitis; Neoplasm metastasis

\section{Introduction}

Cryptococcus is an encapsulated, yeast-like fungus that rarely causes infection in an immunocompetent person. It is ubiquitous in the environment, and the common carriers are pigeon droppings and/or soil contaminated with pigeon excreta. Any organ or tissue could be infected with cryptococcus, although lungs and central nervous system are the most commonly involved. However, cryptococcal infection to localized bone including vertebrae could be possible, although the incidence is very rare. We present the case of a 66-year-old female patient diagnosed as isolated lumbar cryptococcosis mimicking metastatic cancer.

\section{Case Report}

The patient was a 66-year-old female with a history of rectal cancer. She underwent surgery (low anterior resec- tion) for rectal cancer 6 years ago and was treated with adjuvant chemotherapy. Follow-up studies have not revealed any evidence of recurrence for about 6 years. At 5 months before visiting our clinic, back pain developed. At that time, she visited local pain clinics and was treated with medication and epidural block. After a few months later, her symptom aggravated despite every conservative treatment. Then, she received lumbar spine magnetic resonance imaging (MRI) and was referred to our institution. Plain radiographs showed multiple sclerotic lesions at L2 vertebral body (Fig. 1). MRI showed peripheral low and central high signal intensity at L2 vertebral body in T1-weighted image, and irregular enhancement pattern at L1 and L2 vertebral body (Fig. 2). In addition, computed tomography (CT) showed osteolytic lesion in L2 vertebral body (Fig. 3). Based on imaging studies, metastatic tumor was highly suspected. Then, whole body positron emission tomography (PET)-CT, chest CT, and abdominal CT

Received Dec 18, 2014; Revised Jan 8, 2015; Accepted Jan 9, 2015

Corresponding author: Jae Hwan Cho

Department of Orthopedic Surgery, Asan Medical Center, University of Ulsan College of Medicine,

88 Olympic-ro 43-gil, Songpa-gu, Seoul 05505, Korea

Tel: +82-2-3010-3549, Fax: +82-2-3010-8555, E-mail: spinecjh@gmail.com 
were checked to evaluate the disease status of the rectal cancer. PET-CT showed hypermetabolic activity in L1 and L2 vertebral body and no evidence of metastasis in previous surgical bed. There was no evidence of distant metastasis to internal organ or thorax on chest CT and abdominal CT. Lab examinations showed slight elevation of white blood cell (WBC) count $(11,400 / \mu \mathrm{L}$; normal range, $4,000-$ $10,000 / \mu \mathrm{L})$ and erythrocyte sedimentation rate (ESR) (28 $\mathrm{mm} / \mathrm{hr}$; normal range, 0-9 $\mathrm{mm} / \mathrm{hr}$ ). However, C-reactive protein (CRP) was within the normal range $(0.1 \mathrm{mg} / \mathrm{dL}$; normal range, $0-0.6 \mathrm{mg} / \mathrm{dL}$ ). Because she had a mild rhinitis at this time, slight elevation of WBC count and ESR was thought to be reasonable. In that period, excisional biopsy was planned to control metastatic tumors. At the time of operation, the neurologic status of the patient was normal, and systemic symptom such as fever was not observed.

The operation was performed with a combined approach. First, posterior pedicle screw fixation was performed from T12 to L4 posteriorly. Second, anterior

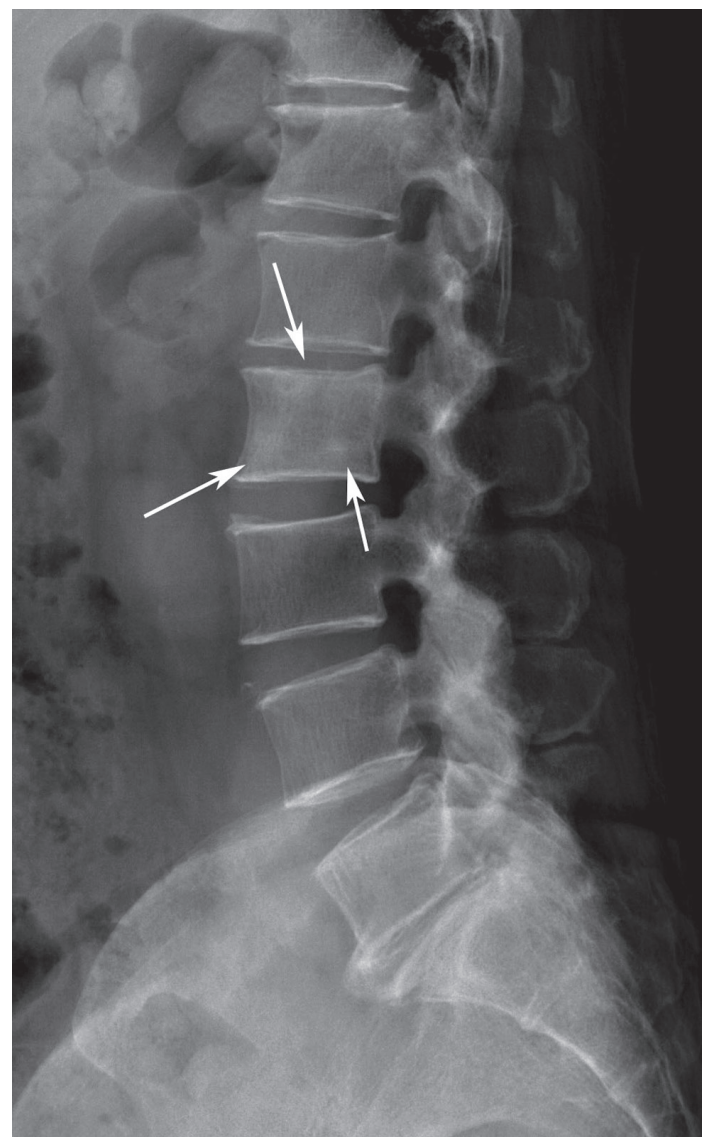

Fig. 1. Preoperative lumbar standing lateral radiograph showing multiple sclerotic lesions at L2 vertebral body (arrows). corpectomy of L2 vertebral body and additional curettage of L1 with expandable cage insertion was performed anteriorly. Definitive pus-like discharge was not found, and soft material was identifiable in the center of L2 vertebral body. We obtained those materials and requested for pathologic examination. Tissue culture study was not done at this time. Final pathologic result showed small yeast-like organisms with Gomori methenamine silver, Fontana Masson, periodic acid-Schiff and Alcian blue stains (Fig. 4). No tumor cells were shown by cytokeratin immunohistochemical staining.

Further studies were performed including blood cultures, cerebrospinal fluid (CSF) study, chest CT, and brain CT to distinguish isolated vertebral cryptococcosis and disseminated cryptococcosis. There was no evidence of lung or brain involvement, and all results were negative including CSF culture, CSF cryptococcal antigen, and
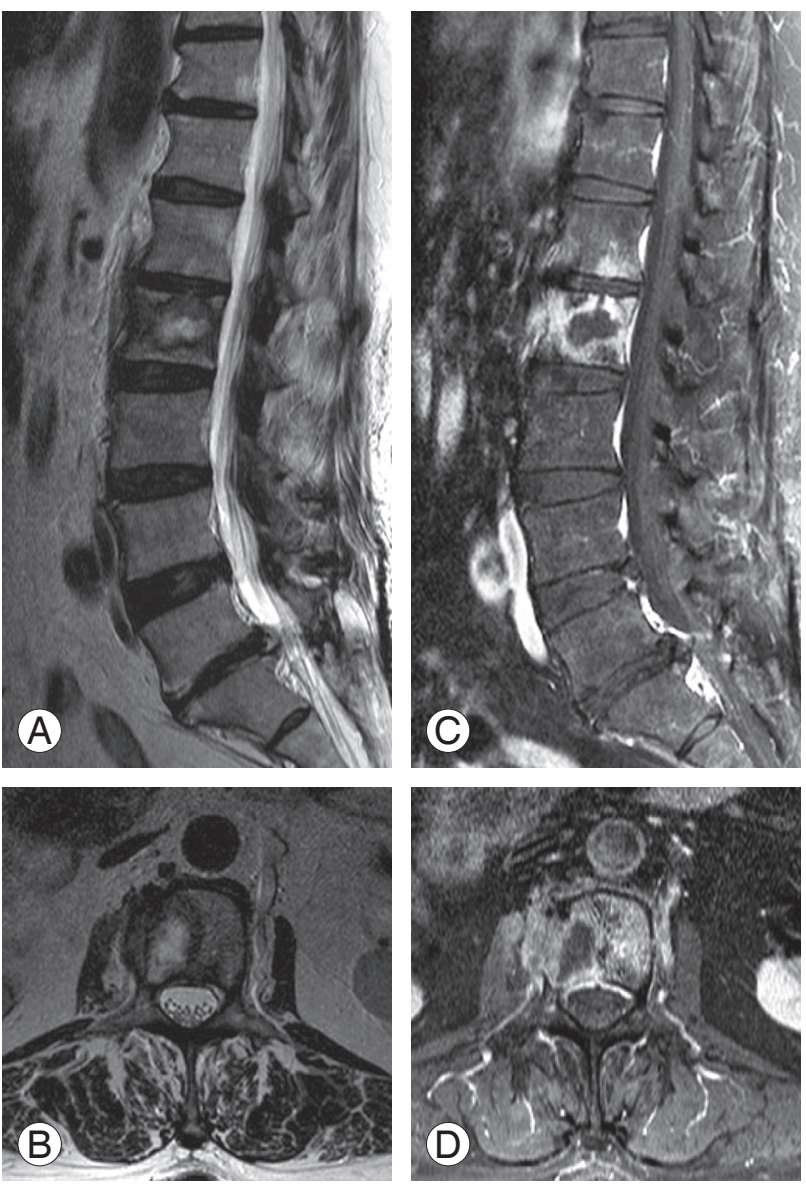

Fig. 2. Sagittal and axial images in magnetic resonance imaging (A, B). T2-weighted images showing peripheral low, central high signal intensity in L2 (C, D). Gadolinium-enhanced images showing mixed signal intensity around $\mathrm{L} 1$ and $\mathrm{L} 2$. 

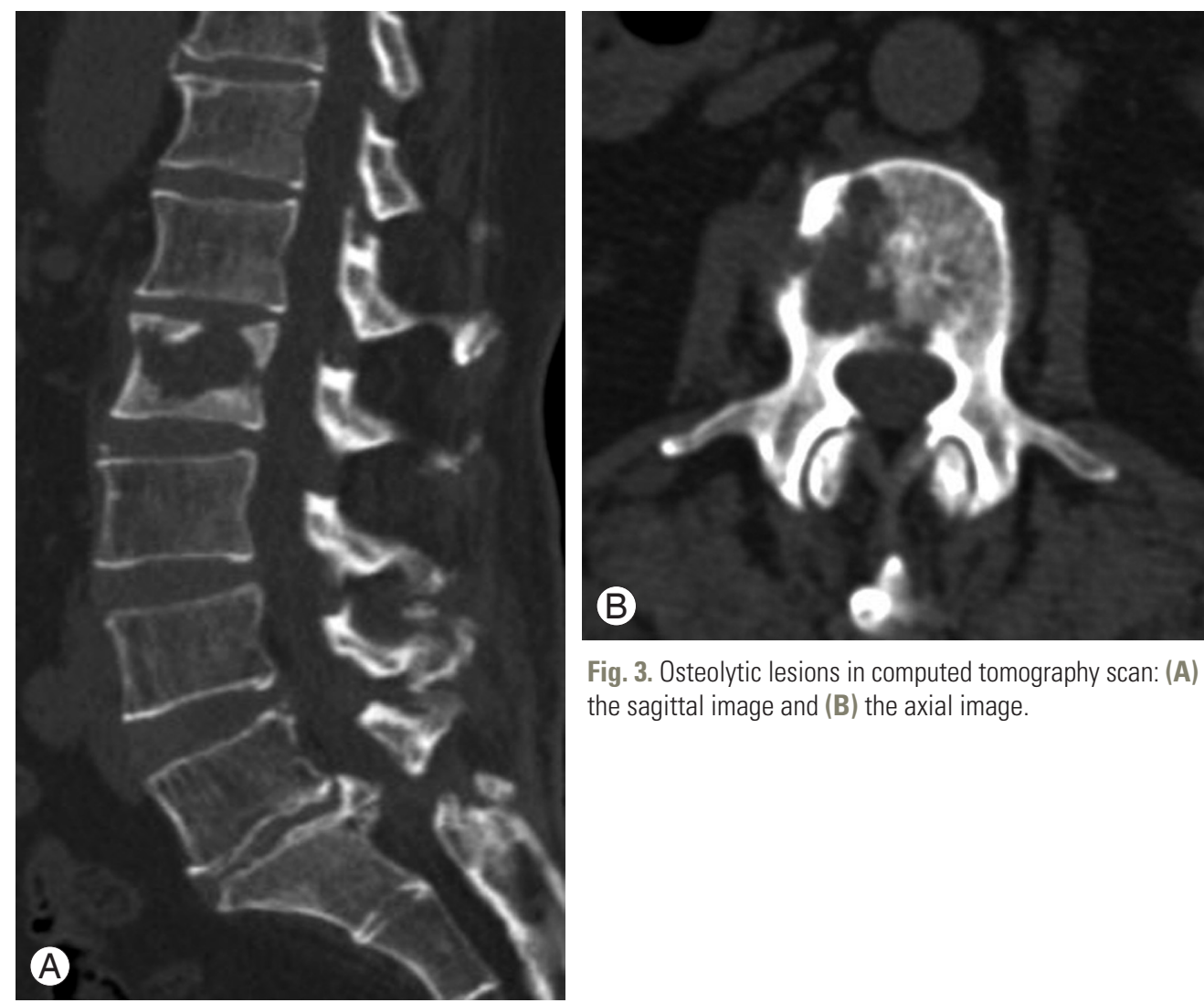

Fig. 3. Osteolytic lesions in computed tomography scan: (A) the sagittal image and (B) the axial image.
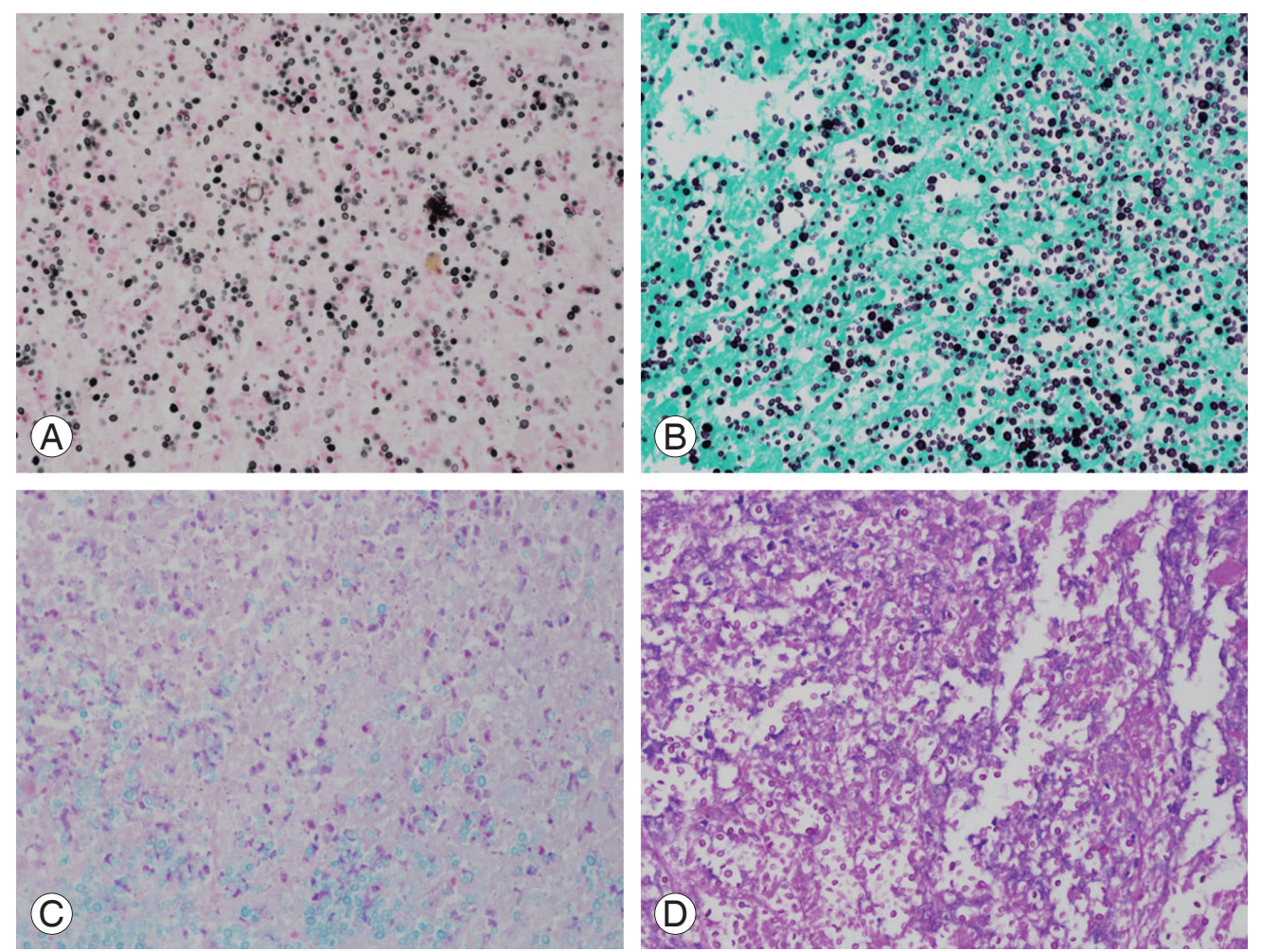

Fig. 4. Pathologic samples with different staining methods. (A) Fontana Masson (×200). (B) Gomori's methenamine silver (×200). (C) Alcian blue (×200). (D) Periodic acid-Schiff (×200). 
CSF pleocytosis. There was no evidence of cryptococcemia on blood culture. Amphotericin B (50 mg/day) and fluconazole ( $800 \mathrm{mg} /$ day) were applied intravenously for about 1 week until disseminated cryptococcosis was ruled out. Then, fluconazole (450 mg/day) was applied orally for about 1 year. At the 1-year follow-up, back pain disappeared without any complications, and no specific abnormal findings were observed in plain radiographs (Fig. 5).

\section{Discussion}

Cryptococcal infection usually occurs in the immunocompromised hosts such as human immunodeficiency virus patients and in patients having an organ transplant or long-term corticosteroid therapy. It is rarely developed in the immunocompetent host. In addition, isolated vertebral involvement is very rare. In fact, it was reported that bone involvement occurred in $5 \%-10 \%$ of disseminated central nervous system infections; however, isolated osteomyelitis was uncommon [1-5]. One study reviewed 47 cases of isolated cryptococcal osteomyelitis from 1974 to 2005 [1]. Forty-one cases had information about underlying conditions. Of those patients, 16 patients (34\%) had no reported underlying conditions, nine patients (19\%) had sarcoidosis, three patients $(6.4 \%)$ had tuberculosis, and four patients (8.5\%) had diabetes mellitus. Other cases were chronic lymphocytic leukemia, cancer, chronic sinusitis, liver transplant, or systemic lupus erythematous, respectively. Thus, the majority of those patients had identifiable immunosuppressive conditions such as sarcoidosis, diabetes, or prolonged treatment with corticosteroids. However, the patient in this case did not show immunosuppressive condition. In addition, no other risk factors for cryptococcal infection were found. She is a housewife, lives in the city (Changwon, Korea) and has never been abroad. It is hard to think that she has contacted the endemic area of cryptococcus. She usually went mountain climbing for exercise; however, this is unlikely to be the cause of the infection.

It was also reported that the vertebral involvement of cryptococcus can mimic tuberculous spondylitis $[6,7]$. Osteomyelitis due to Cryptococcus neoformans typically exhibits lytic lesions with mild or absent periosteal reaction on radiologic examination, as our case does, and that may mimic malignant neoplasms $[8,9]$. However, because
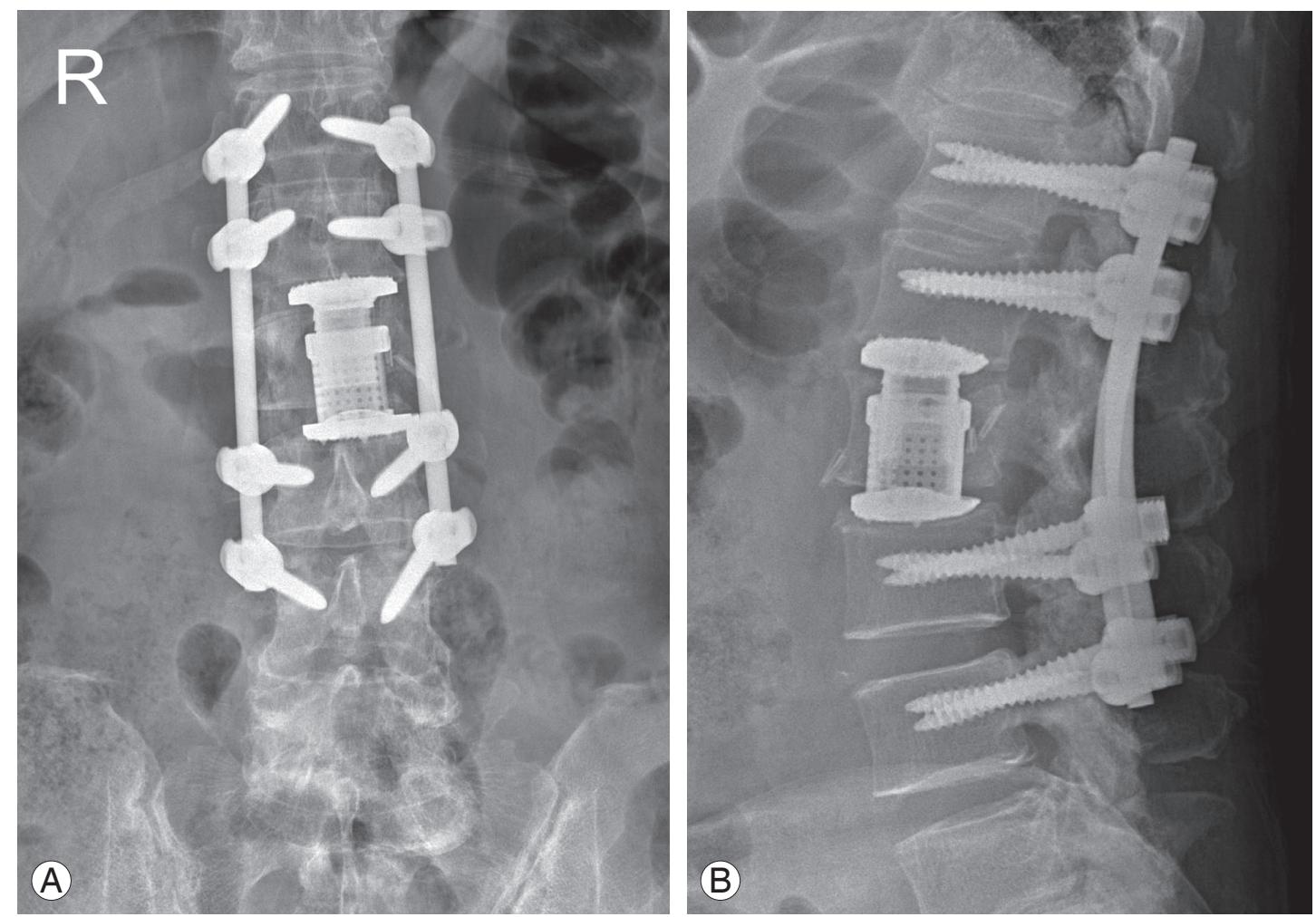

Fig. 5. Plain radiographs obtained in postoperative 1 year visit: (A) the anteroposterior view and (B) the lateral view. 
typical osteolytic lesion was shown in imaging studies and there was a history of rectal cancer, diagnostic possibility other than metastatic cancer was not considered preoperatively. In addition, it was hard to suspect opportunistic fungal infection because the patient had no accompanying illness indicative of immune dysfunction. Interestingly, a few studies have revealed that variable periosteal reactions can be misleading, so lesions could be mistaken for malignant neoplasms $[5,9]$. In our case, multifocal sclerotic reaction was found in $\mathrm{L} 2$ vertebral body in the plain lateral radiograph. This could be one of the misleading factors for the current misdiagnosis. Elevated ESR and WBC count were known to be significant for most cryptococcal osteomyelitis [9]. These findings were also shown in the current case. However, they were not thought to be infection signs because the patient had mild rhinitis symptom without fever at that time. Furthermore, the CRP was in the normal range.

There are two regrettable things in this case. First, it could have been better if percutaneous biopsy was performed preoperatively. However, it was hard to consider possibility other than metastasis. Second, we did not perform the culture study with the tissue obtained during operation, because of the same reason. So, it was impossible to know the specific species of cryptococcus. However, it was estimated as C. gattii rather than C. neoformans. Unlike C. neoformans, C. gattii is not known to be particularly associated with human immunodeficiency virus infection or other forms of immunosuppression. $C$. gattii appears to have a greater propensity to infect immunocompetent humans [10].

In conclusion, isolated cryptococcal vertebral osteomyelitis in immunocompetent patient occurs as a very rare incidence. However, it can mimic metastatic cancer as in the current case. For this reason, cryptococcal osteomyelitis should be considered as one of the differential diagnosis in patients who are suspected of isolated vertebral metastatic lesion. In addition, it could be better to do intraoperative culture study when excision of metastatic tumor is performed. If cryptococcal osteomyelitis is confirmed, antifungal therapy alone could be enough to cure.

\section{Conflict of Interest}

No potential conflict of interest relevant to this article was reported.

\section{References}

1. Al-Tawfiq JA, Ghandour J. Cryptococcus neoformans abscess and osteomyelitis in an immunocompetent patient with tuberculous lymphadenitis. Infection 2007;35:377-82.

2. Behrman RE, Masci JR, Nicholas P. Cryptococcal skeletal infections: case report and review. Rev Infect Dis 1990;12:181-90.

3. Burch KH, Fine G, Quinn EL, Eisses JF. Cryptococcus neoformans as a cause of lytic bone lesions. JAMA 1975;231:1057-9.

4. Fialk MA, Marcove RC, Armstrong D. Cryptococcal bone disease: a manifestation of disseminated cryptococcosis. Clin Orthop Relat Res 1981;(158):219-23.

5. Zach TL, Penn RG. Localized cryptococcal osteomyelitis in an immunocompetent host. Pediatr Infect Dis 1986;5:601-3.

6. Gurevitz O, Goldschmied-Reuven A, Block C, Kopolovic J, Farfel Z, Hassin D. Cryptococcus neoformans vertebral osteomyelitis. J Med Vet Mycol 1994;32:315-8.

7. Gupta SK, Chhabra R, Sharma BS, Das A, Khosla VK. Vertebral cryptococcosis simulating tuberculosis. Br J Neurosurg 2003;17:556-9.

8. Witte DA, Chen I, Brady J, Ramzy I, Truong LD, Ostrowski ML. Cryptococcal osteomyelitis: report of a case with aspiration biopsy of a humeral lesion with radiologic features of malignancy. Acta Cytol 2000;44:815-8.

9. Qadir I, Ali F, Malik UZ, Umer M. Isolated cryptococcal osteomyelitis in an immunocompetent patient. J Infect Dev Ctries 2011;5:669-73.

10. Dixit A, Carroll SF, Qureshi ST. Cryptococcus gattii: an emerging cause of fungal disease in North America. Interdiscip Perspect Infect Dis 2009;2009:840452. 